\title{
La herida original de las políticas de inmigración. A propósito del lugar de los derechos humanos en las políticas de inmigración
}

\author{
JAVIER DE LUCAS \\ Universidad de Valencia
}

\begin{abstract}
RESUMEN. El artículo plantea algunas condiciones que permitan superar las dos posiciones en las que parece alternativamente preso el debate actual sobre la inmigración: la visión instrumental, torpemente pragmática, y la «humanitaria», anclada en la conmiseración o la piedad. Para alcanzar la dimensión política es preciso superar un análisis de los flujos migratorios erróneo, el que está en la base de ambas posiciones. Y si se supera esa visión, se superarán los actuales instrumentos jurídicos de política de inmigración, que no son acordes con el reconocimiento pleno y la garantía de los derechos humanos fundamentales, pero tampoco con el necesario reconocimiento de la condición de sujeto del espacio público a los inmigrantes que residen establemente, es decir, con las exigencias de una democracia plural e inclusiva, que es el reto, pero también la oportunidad que nos ofrece otro análisis de la inmigración, que recupere la dimensión política profunda de este fenómeno, en el orden interno y en el internacional.
\end{abstract}

\begin{abstract}
In this article I want to discuss some principles which may go beyond the two mainstream perspectives of the immigration debate nowadays: the instrumental one, focusing in pragmatic considerations and the humanitarian one, related to compassionate values. Both perspectives affect the analysis of immigration flows in a wrong way. To improve the terms of this debate it is necessary to rebuilt the current legal actions in immigration policy which questions the basis for the recognition of human rights and the belonging and participation of denizens in the public sphere. The recognition of this principles is essential in a pluralistic and inclusive democracy and enriches the analysis of immigration from a political perspective in the domestic and international sphere.
\end{abstract}

\section{Inmigración: el lugar de los derechos humanos en el debate público}

Uno de los aspectos más llamativos, a la vez que significativos, del debate actual acerca de las políticas de inmigración es el que afecta al reconocimiento de los derechos - humanos y fundamentales ${ }^{1}$ - de los inmigrantes. Es llamativo

${ }^{\prime}$ Doy por suficientemente conocida la distinción, que sigo en los términos en los que la ha explicado entre nosotros A. E. Pérez Luño. 
porque resulta sorprendente la escasa importancia que se atribuye a la cuestión. Es significativo porque me parece que ilustra muy bien las razones del déficit de nuestras políticas de inmigración, empezando por las de nuestra propia mirada sobre el fenómeno migratorio, tal y como se produce hoy y muy concretamente en dirección a los países de la UE.

La escasa atención prestada a la cuestión de los derechos humanos de los inmigrantes en el debate público acerca de las políticas de inmigración (más allá de la retórica que hace de ellos una referencia obligada en cualquier discurso, en cualquier preámbulo normativo, en las ruedas de prensa que acompañan a las citas europeas, es decir, en el terreno declamativo), o, dicho de otra manera, el hecho de que, una vez más parafraseando a Dworkin, no nos tomemos en serio esos derechos, puede explicarse, a mi juicio, entre otras razones, por las siguientes.

La primera, porque las cuestiones relativas a esos derechos no parecen directamente relevantes en la definición de las tres piezas clave de toda política de inmigración (control de los flujos, gestión de la presencia de los inmigrantes en el propio país, relaciones con los países que generan «nuestra» inmigración). Y eso es así porque se da por supuesto que los derechos de los inmigrantes son algo previo a esas cuestiones, ya que están garantizados. ¿Cómo podría ser de otra manera, si reconocemos en todas nuestras Constituciones y en los instrumentos jurídicos-marco (como el Convenio europeo de 1950) los derechos humanos universales? ¿Acaso no hemos ratificado todos los Estados de la UE la Declaración del 48 y los Pactos del 66? Por eso, se nos pide que no repitamos lo obvio y hagamos política en serio. La política de inmigración no sería, conforme a este planteamiento, una cuestión de derechos, que se deben dar por supuestos como en todas las políticas públicas. No es un problema de derechos, pues, sino en todo caso de instrumentos adecuados de desarrollo de los mismos. Pero lo cierto es que no es así, y que ni en la gestión de los flujos (reducida a policía de fronteras), ni en la gestión de la presencia de los inmigrantes en los países de recepción (en la que impera la consideración de las necesidades de mercado y la lógica del orden público, como elementos reales de la proclamada integración, junto a un cada vez mayor prejuicio asimilacionista), ni en la relación con los países de origen (dominada a su vez por la obsesión de asociarlos en las funciones de policía de fronteras, tanto en salida como en repatriación, y adecuación de cupos al mercado laboral de los países de destino) priman los derechos humanos.

Por eso, precisamente, la segunda razón, cercana al pragmatismo habitual: i «no es un problema de derechos. Se trata de medidas políticas», nos aseguran. $\mathrm{Y}$ con ello, como veremos, se quiere decir que la inmigración es una cuestión de números, de estadísticas, sobre todo de dos. En primer lugar, de las estadísticas que dictan la adecuación de la inmigración al mercado laboral, que es la dimensión básica de nuestra mirada sobre la inmigración (y por eso la obsesión con los cupos, las cuotas o contingentes como criterio mágico de 
esas políticas: determinar que vengan los que necesitamos, ni más ni menos), es decir, la inmigración en términos de la lógica del beneficio propia del mercado. En segundo lugar, las estadísticas que nos muestran el umbral de tolerancia del orden público (la relación de la inmigración con el ejército de reserva de la delincuencia, el incremento insostenible de los delitos frente a los que la opinión pública es más sensible, la relación de la presencia de inmigrantes con la sostenibilidad de nuestro sistema penitenciario, con la parálisis de los tribunales), es decir, la inmigración como cuestión de seguridad. Mercado y seguridad, al fin de cuentas los dos ejes de la política en serio, de la propia de aquellos que contraponen la ética de la responsabilidad a la de los principios, a la abstracción de las discusiones sobre los derechos que pueden permitirse quienes no han de tomar las decisiones. Y no digamos en el mundo post-11S, en el que la lógica política al uso parece la de la obsesión securitaria, que propicia la reinstauración más torpe y maniquea de la concepción schmittiana. La política según la lógica del amigo/enemigo, que exacerba hasta la simplificación el mensaje hobbesiano, ahora con el terrorismo fundamentalista como piedra de toque que permite estigmatizar apriorísticamente la inmigración y no digamos el fobotipo de la misma, los inmigrantes ilegales, identificados con el retrato-robot del fundamentalista islámico que viene a poner en peligro nuestra democracia, nuestros derechos humanos universales. Un enemigo frente al que ya ha dejado claro Sartori que la ciudadanía y los derechos son una respuesta que no debemos conjugar.

Frente a esa dosis de realpolitik, lo cierto es que, en términos de justicia y/o legitimidad, la discusión del reconocimiento de derechos humanos -pero también de los derechos fundamentales- a los inmigrantes, apenas merecería unos párrafos, habida cuenta de la obviedad de la respuesta. Por supuesto, no me refiero al estado de la cuestión en el Derecho Comparado, en el que, lamentablemente, la regla es la discriminación pretendidamente justificada a propósito del reconocimiento de los inmigrantes como titulares de esos derechos. No me refiero al reconocimiento y garantía de esos derechos en los instrumentos jurídicos de política de inmigración, incluso en los del Occidente civilizado (los de la Unión Europea, por más señas) que hacen suyo el santo y seña de los derechos universales como condición de legitimidad y estrella polar de sus ordenamientos jurídicos y de su orden social. Hablo de la pregunta que nos ocupa: itienen, deben tener, los derechos humanos y fundamentales de los inmigrantes un lugar central en nuestras políticas de inmigración? Y si de hecho no es así, ¿cuál es la razón?

Inșisto: obviamente, no hay ninguna razón, quiero decir, una buena razón -razón moral, razón de legitimidad (si se me permite hablar en estos términos de forma gruesa)-, que justifique la falta de reconocimiento o la discriminación en el reconocimiento (tampoco en la garantía) de los derechos humanos universales, pues va de suyo que, si así los afirmamos, la condición de inmigrante es irrelevante. Pero me parece que tampoco precisamos mucho esfuerzo para 
llegar a la conclusión de que tampoco hay buenas razones de ese orden para justificar o legitimar la discriminación en el reconocimiento y garantía de los derechos fundamentales de los inmigrantes: ni respecto a los derechos civiles, ni a los económicos, sociales y culturales podemos presentar como justificada la discriminación en el reconocimiento de los inmigrantes como titulares de esos derechos. La condición de inmigrante no es una buena razón para justificar discriminación. La única barrera se alzaría a propósito de los derechos característicos de la ciudadanía, reservados a los miembros de la sociedad política, de la que no forman parte los inmigrantes precisamente por su condición de tales. Y es cierto que en el debate acerca de las políticas de inmigración esta aproximación a la ciudadanía ha adquirido -0 reforzado- un carácter de postulado. No podemos tratar a los inmigrantes como si fueran ciudadanos, cuando es obvio (como se trataba de mostrar, claro) que no lo son.

Pues bien, precisamente lo que llama la atención es que, pese a tal cúmulo de obviedades, los instrumentos jurídicos de política de inmigración de los países de la Unión Europea (de España, desde luego), por referirnos a nuestro contexto más inmediato $\mathrm{y}$, lo que es más grave, la opinión pública de esos países, dan por obvio precisamente lo contrario de lo que dicen sostener y realizan una discriminación en el reconocimiento y en la garantía efectiva de derechos humanos y fundamentales de los inmigrantes y, además, una discriminación pretendidamente justificada. Debo precisar que me refiero a todos esos derechos, también a los políticos, porque, a mi juicio, resulta grave la mayor obviedad, la presentación como justificada si no obligada - casi en términos de «natural»- de la discriminación (que en realidad es subordinación) en el reconocimiento de derechos políticos a los inmigrantes, lo que repercute directamente en su reconocimiento como sujetos del espacio público y es una de las razones del déficit de construcción de éste en términos de una democracia plural e inclusiva, algo que, a mi juicio, no han sabido ver suficientemente quienes tratan de abrir la tradición liberal democrática a ese nuevo modelo, el que exige la gestión democrática de las sociedades multiculturales, como Rawls - desde dentro de esa tradición - o Habermas.

En particular, llama la atención el cimismo con el que se presenta un mensaje que, por mucho que se esconda tras la apariencia de lógica jurídica (técnicojurídica, gustan de decir) a duras penas puede ocultar su carácter de pieza angular de la lógica que alimenta la extensión de los «procesos de exclusión», tal y como ha mostrado Robert Castel, una lógica que golpea en particular a los inmigrantes, un proceso de vulnerabilidad cuyo primer elemento es la precarización del trabajo y las políticas que tienen como resultado el cierre de los cauces que permitían lo que Hoggart ${ }^{2}$ denominara la porosidad progresiva entre las viejas dos clases antagónicas, los mecanismos transversales que permitían un puente: la propiedad de la vivienda, la asistencia contra los riesgos sociales (enfermedad, vejez, desempleo), el acceso a la educación,

\footnotetext{
${ }^{2}$ R. Hoggart, 1970.
} 
etc. Todo eso significa, como ha advertido, por ejemplo, Gaullier, la desestructuración de los ciclos de vida social, configurados en gran medida por la sucesión de las etapas laborales y que conducen a la gestión del tiempo de ocio y de retirada de la vida activa también como factores de estabilidad social; eso es tanto como decir que las consecuencias no se reducen al ámbito laboral, que alcanzan no sólo a la integración social que el trabajo produce, sino a la estabilidad social misma, a la integración social en un orden en el que estén suficientemente garantizados las necesidades y derechos básicos. Se crea así - la expresión es de Castel- una especie de «no-man's land social» que, además, comienza a heredarse; se potencia un proceso de vulnerabilidad que amenaza con extender de forma insoportable los estados de exclusión. Como advierte Walzer ${ }^{3}$, son ellos quienes «participan sólo en una mínima parte en el bienestar de sus países, soportan el peso de la crisis económica, son expulsados de las mejores escuelas y de los mejores puestos, llevan por todas partes los signos de los perdedores. Así reproducimos las exclusiones internas del mundo antiguo: los sin-derechos, los sin-poder, los parados, los marginados». Que se sostenga frente a ello el mito de la exclusión justa (justificada al menos como inevitable, como exigida por las reglas de la «racionalidad económica») sólo pueden entenderse desde el cinismo. Y eso es precisamente lo que hoy se pretende con los inmigrantes.

Me refiero al mensaje de las políticas de inmigración cuando tratan de justificar su exclusión, su sometimiento a condiciones de vulnerabilidad ${ }^{4}$ con criterios injustificables, como la lotería del nacimiento, criterios que chocan con la fe en la universalidad y en la libertad y autonomía como único criterio de juicio. La disputa en torno al derecho a la salud de los inmigrantes es sólo un botón de muestra, como otros tantos: no se habla del derecho a la salud, sino de qué tipo de prestaciones sanitarias y hasta dónde hay que garantizar a los inmigrantes (a diferencia de los ciudadanos), casi en términos de cuáles son los «costes de mantenimiento» asumibles para mantener los beneficios (estaría uno tentado de decir los costes de atención veterinaria), es decir, la que se presta a un instrumento o un animal para que funcione. Lo mismo sucede con la «rebaja» en el reagrupamiento familiar, que no es un derecho de todos los miembros de la familia, sobre todo si se habla de integración de los inmigrantes como objetivo prioritario, sino un instrumento de política de inmigración en la medida en que la inmigración «familiar» es percibida como un creciente y amenazante cauce de entrada de inmigración.

\footnotetext{
${ }^{3}$ Walzer, 1992 , p. 100.

${ }^{4}$ Qué mayor vulnerabilidad que no tener derechos, que no sólo reaparecen (sabemos que las leyes permiten, posibilitan incluso que se vuelva a «caer en la ilegalidad»), sino que evidentemente se transmiten entre ellos, se heredan, porque les acomunan, acompaña a su propia condición de inmigrantes.
} 


\section{Un debate entre el humanitarismo y la eficacia que olvida los derechos}

A mi juicio, el hecho de que los derechos de los inmigrantes no sean cuestión prioritaria en la «agenda política» responde a nuestra mirada, nuestra comprensión del fenómeno de la inmigración, una mirada que se refleja en los términos del debate: hoy, en el debate público sobre la inmigración, seguimos anclados en una alternativa deleznable, entre la conmiseración (la piedad, o, para que no quede tan mal, el discurso «humanitario») y el pragmatismo utilitarista más ralo, el mercantilismo (claro que peor es el punto de vista excluyente, xenófobo y/o racista). Para decirlo más exactamente, incluso el motto humanitario empieza a ceder ${ }^{5}$. Sin duda, influye la «filosofía» del 12 de septiembre en ese recrudecimiento de la prevención, el discurso de la «barca llena», que se impone a las razones humanitarias. Porque, como he señalado, crece hasta alcanzar casi el grado de obsesión el viejo diagnóstico del «conflicto de civilizaciones», concretado en el fobotipo del inmigrante de la ribera sur, identificado en términos acríticos y generalizantes - pero sumamente eficaces ante la opinión pública- como fundamentalista islámico y terrorista, frente a quien (insisto, Sartori dixit) no vale el discurso de la ciudadanía, la democracia y los derechos, por su carácter inasimilable e incompatible (una argumentación que pasa desde el diferencialismo cultural - que es el mensaje del nuevo racismo- a la justificación de discriminaciones inaceptables en su status jurídico y político).

Lo peor de esa mirada errónea sobre la inmigración, de cuyos presupuestos me ocuparé enseguida, son dos consecuencias en las que he intentado insistir en otros trabajos.

En primer lugar, la contaminación de la lógica de las libertades y del Estado de Derecho. Quiero decir que el mensaje que envían los instrumentos jurídicos de política de inmigración que responden a esos supuestos es en realidad incompatible con los principios mínimos del garantismo que emanan de aquéllas. Porque nuestras leyes de inmigración, por decirlo sin mayores precisiones, envían a nuestros ciudadanos (antes incluso que a los inmigrantes) este mensaje: está justificada la discriminación en el reconocimiento de los derechos humanos y fundamentales de los inmigrantes (no digamos en los derechos legales, sin más), su condición de sujetos jurídicos (y sujetos del espacio público) de segundo orden, no como nosotros, el empeño en la segmentación de status que se convierte en la creación de status de infraciudadanía, precisamente por su condición de inmigrantes.

\footnotetext{
${ }^{5}$ Un sondeo considerado como fiable, el sondeo semanal de la SER de 20 de mayo de 2002, ofrece ya un retrato preocupante acerca de ese decaimiento del espíritu humanitario: más del 60 por 100 de los españoles creen en la asociación entre inmigración y delincuencia, el 75 por 100 la considera excesiva, más del 70 por 100 la percibe como un problema en el que lo más importante es adoptar medias para limitar la llegada de inmigrantes.
} 
$\mathrm{Y}$ en segundo término, los instrumentos de políticas de inmigración nos devuelven una mirada sobre la inmigración, una comprensión de la misma en términos instrumentales, unidireccionales, reductivos. Lo que es peor, nuestras políticas de inmigración niegan la centralidad del fenómeno de la inmigración como cuestión política, incluso como la cuestión política precisamente en sociedades multiculturales, al menos como oportunidad para pensar de otro modo la política, para reconsiderar el modelo de democracia propia de sociedades que ya no pueden seguir sosteniendo la presunción de homogeneidad y con ella el modelo del consenso en los que se basa nuestro modelo de democracia liberal. Porque al negar la realidad de los flujos migratorios, su dimensión global y compleja, los reducen a esas cuestiones estadísticas en los que tratan de resolverlos. Y olvidan que la inmigración hoy es sobre todo una oportunidad para pensar cuáles son las razones de pertenencia, cuál es la justificación del vínculo social y político. Una oportunidad para reconocer esa pertenencia como un bien primario que es la ciudadanía, que no puede reservarse - sin reconocer que con ello se convierte en privilegio (así lo denuncian Balibar, Ferrajoli) y aun en privilegio sin justificación posible - a los nacionales, a quienes fueron beneficiados por la lotería genética o, mejor, geográfica, en definitiva, pese a lo que presume la concepción de la democracia liberal, a quienes forman parte de ese nosotros que no puede no ser etnocultural. En todo caso, estoy convencido de que para construir una política de inmigración adecuada a las exigencias del Estado de Derecho y a la vez a las condiciones reales, incluso esas razones piadosas son malas razones, porque posponen una vez más el debate acerca de lo que significa hoy, para nosotros, la inmigración, y lo que debemos hacer.

En lo que sigue, trataré de exponer algunos elementos que, a mi juicio, ponen de manifiesto las razones del déficit de esa comprensión de la inmigración que muestran las políticas de inmigración y propondré algunos criterios de corrección. Unos y otros son en buena medida coincidentes con los intentos de introducir en este ámbito la justicia como criterio prioritario, antes que el mercado, tal y como lo ha señalado con acierto Ricard Zapata ${ }^{6}$.

\section{Por qué los inmigrantes no son sujetos universales de derechos} o de cómo la distinción hace la luz y crea las categorias jurídicas en la inmigración

Si hay una metáfora que nos permita entender la mirada de nuestras políticas de intmigración ésa es la fórmula «sin papeles», o, más brutalmente, la noción de ilegales. Precisamente porque nos muestra sin tapujos cómo hemos creado

${ }^{6}$ Zapata, 2002, pp. 37-75, donde sigue las tesis de Carens, incluso en su evolución desde un universalismo tout court a una posición más pragmática, como la de Withol der Wenden, para proponer un modelo que incorpore criterios de justicia, según la propuesta de «justicia local» de Walzer y Elster, que hace suya. 
ese peculiar sujeto (no-sujeto) al que calificamos como inmigrante. A los inmigrantes les falta, de hecho, que tomemos en serio su condición de sujetos del derecho a tener derechos. Y en primer lugar, del derecho en que consiste la condición de inmigrante, que es por otra parte la del ser humano mismo, la libertad de movimiento, el derecho a desplazarse libremente que los clásicos españoles como Vitoria o Suárez, siguiendo las tesis del estoicismo, entendieron como primer derecho propio de un mundo entendido en su dimensión cosmopolita como communitas omnium gentium (y no un derecho de libre desplazamiento cuyo titular son los imperios o las grandes compañías comerciales, como sostuvo en realidad el abogado Grotius) ${ }^{7}$. Es sabido que en el Derecho positivo (internacional ni comparado) no se reconoce ese derecho, pues el derecho de libre deambulación proclamado en la Declaración del 48 no lleva consigo la libertad de asentamiento, que queda siempre sujeta a una cláusula de restricción que reenvía a la competencia de cada Estado para determinar las condiciones. Pero lo que es más grave es la negación de la condición de sujeto de derechos del inmigrante como tal, una negación que se produce como consecuencia de la restricción del concepto mismo de inmigrante, que, contra lo que dicta el sentido común y la razón, no es el sujeto, el ser humano que ejerce su libertad de movimiento, con el propósito que determine su libre autonomía, sino sólo el que se mueve dentro de los límites del inmigrante económico, el inmigrante trabajador, que es el verdadero y buen inmigrante. Aunque se admitan categorías subordinadas a ésta (el inmigrante por reagrupación familiar, el inmigrante arrepentido o denunciante de los traficantes y de los malos inmigrantes), la categoría central de inmigrante, la que tiene relevancia jurídica, es ésa. Veamos por qué.

En efecto, nuestra mirada sobre la inmigración, como también sobre la multiculturalidad, se basa paradójicamente en la negación de su objeto ${ }^{8}$, pues consiste en negar al inmigrante como tal inmigrante, es decir, alguien cuyo proyecto - plural - puede ser perfectamente tratar de quedarse en el país de recepción, al menos durante un período estable que tampoco significa necesariamente (menos aún en los tiempos de la globalización) quedarse para toda la vida, al menos en el proyecto de la primera generación.

Nuestra creación de la noción de inmigrante está presidida por la negación de la posibilidad misma de ser inmigrante de verdad, esto es, libre en su proyecto migratorio -el que sea-, basado simplemente en la libertad de circulación. En lugar de aceptar esa posibilidad o, al menos, abrirla, se extranjeriza al inmigrante, se le estigmatiza, congelándolo en su diferencia, como distinto (ex-

\footnotetext{
2 ${ }^{7}$ Las consecuencias de esta diferencia fundacional en el propio concepto de Derecho internacional público, pese a que pasa por un tópico, apenas son destacadas en el ámbito de la Filosofía moral, jurídica y política, con raras excepciones (Pérez Luño, Fernández Buey) o en el propio Derecho internacional (Ramón Chornet). A mi juicio, quizá ha sido Sánchez Ferlosio quien ha sabido mostrarlas con mayor claridad en su Esas Indias malditas y olvidadas.

${ }^{8}$ He tratado de mostrarlo ha propósito de la multiculturalidad, recientemente, en De Lucas, $2002 \mathrm{~b}$.
} 
tranjero) y sólo como trabajador útil en nuestro mercado formal de trabajo aquí y ahora. Por eso, se le imponen condiciones forzadas de inmigración, supeditadas al interés exclusivo e instrumental de la sociedad de destino, que sólo le necesita como mano de obra y sujeta a plazo.

Como he tratado de mostrar en otras ocasiones, ése es un modelo de gestión de la inmigración construido a base de la creación de distinciones pretendidamente científicas pero de enorme trascendencia normativa y, sobre todo, maniqueas, como han mostrado, por ejemplo, Castles o Bauböck. Lo más grave es que esas categorías clasificatorias, pese a su pretendida objetividad, no responden a la realidad, no se adecuan a ella $\mathrm{y}$, por tanto, difícilmente pueden servir como instrumentos eficaces para gestionarla. Lo que es aún peor, ignoran la realidad, pues se empeñan en negarla, en desconocerla. Así, distinguen entre buenos y malos inmigrantes, es decir, entre los que se ajustan a lo que nosotros entendemos como inmigrantes necesarios (adecuados a la coyuntura oficial del mercado formal de trabajo ${ }^{9}$, asimilables culturalmente, dóciles) y los demás, que son rechazables, bien por delincuentes (cometen actos delictivos, comenzando por entrar clandestinamente en nuestro país, lo que evoca connivencia con las mafias), bien por imposibles de aceptar (porque desbordan nuestros nichos laborales o son inasimilables): por una u otra razón, constituyen el ejército de reserva de la delincuencia y, rizando el rizo de la argumentación, generan racismo y xenofobia contra los inmigrantes buenos. Aún más. Como ha criticado Castles, buena parte de las políticas contemporáneas de inmigración ha elaborado una tipificación más eficaz, más «científica», la que permite distinguir entre verdadera y falsa inmigración.

La falsa, la inmigración «forzada» - como si la otra fuera puramente libre-, es decir, las manifestaciones clásicas de refugio y asilo, junto a los fenómenos más recientes que calificamos como desplazamientos masivos de población, característicos de quienes huyen de catástrofes de todo tipo, desde las naturales -terremotos, hambrunas, inundaciones, sequías - a las sociales - guerras civiles, conflictos étnicos, religiosos, etc.-. Esta segunda clase, la falsa inmigración, además, tiene hoy una cómoda y - para nosotros - rentable etiqueta: «lo humanitario», de forma que podemos olvidarnos de élla como un asunto a gestionar en el ámbito de políticas de inmigración, salvo para vigilar que ningún inmigrante tout court (el económico) intente «colarse», utilizando fraudulentamente esta segunda vía.

Por el contrario, para asegurarnos cuándo nos encontramos ante la primera, la verdadera inmigración que es, claro, la económico-laboral, la de los trabajadọres, se les impone un corsé diseñado según el viejo modelo del Gastarbeiter, el guest worker, el trabajador invitado, que es, sobre todo, extranjero. Esa figura significa en primer lugar eso, que no es un inmigrante, porque no se quiere aceptar la posibilidad de que venga aquí en otra calidad que la de trabajador; aún menos, de trabajador dentro de un cupo predeterminado.

\footnotetext{
${ }^{9}$ Cf. Martiniello, 2001.
} 
Frente al Gastarbeiter no hay, no puede haber, voluntad de integración, porque no se acepta la posibilidad de que pueda aspirar a quedarse establemente (aunque no sea de forma definitiva, insisto). No hay integración, que supone aceptar que el inmigrante es parte activa en un proceso bidireccional que involucra en el cambio también a la sociedad de acogida, como muestra el exabrupto del alcalde de El Ejido, en plenos acontecimientos racistas del $2000{ }^{10}$ : «a las siete de la mañana, todos los inmigrantes son pocos; a las siete de la tarde, todos sobran». Por eso, una condición tan necesaria del proceso de integración como el ejercicio del derecho de reagrupamiento familiar, no es reconocida como tal, sino como un problema, como una vía no deseada de entrada de seudoinmigrantes (pues sólo son familiares del trabajador, del verdadero inmigrante, que es el trabajador). Por eso no se plantea la prioridad de las condiciones de residencia estable o de verdadera libertad de circulación en los dos sentidos. Por eso la insistencia en que los derechos que corresponde reconocer son sólo los derechos humanos universales y aun estos fuertemente restringidos. Por eso, lo inconcebible de pensar al inmigrante como posible ciudadano ${ }^{11}$.

Es decir, que la prioridad es, ante todo, entender que la inmigración que recibimos no es necesariamente (no lo es, de hecho), lo que nosotros egoístamente queremos recibir para nuestro beneficio y comodidad. La prioridad es cambiar nuestra mirada sobre la inmigración que nos llega y va a seguir llegando, que no es, aunque nos empeñemos, la herramienta dócil cuya presencia se justifica si y sólo si llenar el hueco laboral y demográfico que nosotros hemos decidido, permaneciendo invisible y dejando inalterada a la sociedad de destino. Para eso, además, hay que comprender que no existe el inmigrante, como tampoco la sociedad de acogida: sólo el antropólogo de guardia, el Sr. Azurmendi, políticamente incorrecto pero bien sentado en su cargo, se atreve a hablar de «el inmigrante» y a pontificar con generalizaciones sobre la falta de cultura de trabajo y de disposición para la democracia y los derechos humanos de «los inmigrantes». Los inmigrantes, además de muy diferentes por sus necesidades, su procedencia, su situación, su cualificación (sí, cualificación), albergan proyectos plurales, $y$, entre ellos, el de quedarse. Por eso no vale como patrón de política migratoria el modelo del Gastarbeiter: inmigrar es algo más, en la mayoría de los casos, que ir a hacer los trabajos que necesita la sociedad que los reclama. En muchos de esos proyectos, y desde luego, en los de las generaciones posteriores, el objetivo es convivir establemente, al menos durante un período importante. ¿Estamos dispuestos a aceptarlo? ¿Nos preparamos para ello? Creo que no.

\footnotetext{
${ }^{10}$ Ninguneados ahora desde algunos pretendidos análisis científicos (Azurmendi) y desde el Gobierno español, y paradójicamente destacados por el Observatorio Europeo de Racismo y Xenofobia de Viena como los más graves acontecimientos racistas acaecidos en Europa: cf. el análisis que dedica el EUMC en el núm. 8 de su boletín Equal Voices, de 2002, «El Ejido revisited», Equal Voices, pp. 23-28.

${ }^{11}$ Es lo que lo señalan, por ejemplo, Bauböck (2001), o Castles y Davidson (2000).
} 
No lo estamos porque seguimos pensando que la inmigración es una cuestión de mano de obra y orden público, y nuestra respuesta se basa, pues, en el modelo del guardia de tráfico, en la lógica policial e instrumental/laboral. Lamentablemente, en España y todavía en la UE (aunque hay atisbos de cambio) el modelo de gestión de la inmigración consiste en eso, policía de tráfico y adecuación de contingentes. Pero eso no es política de inmigración. No lo es porque ignora la realidad de la inmigración (insisto, mucho más compleja que lo que nos resulta cómodo imaginar) y no lo es porque resulta incoherente con los principios de legitimidad que aseguramos defender cuando, por ejemplo, hablamos de patriotismo constitucional, si se toma en serio la Constitución.

\section{La universalidad de los derechos exige el reconocimiento de todo otro como otro: la inclusión}

El primero de los derechos humanos, como explicaba $\mathrm{H}$. Arendt, es el derecho a tener derechos, es decir, al reconocimiento como persona, como miembro de la comunidad jurídica y política. En realidad, si lo pensamos por un momento, el alcance de la tesis de la universalidad de los derechos humanos es precisamente éste, el que todos los seres humanos sean reconocidos como sujetos, $\mathrm{y}$ eso no porque se universalice un modelo homogéneo, sino precisamente desde su carácter insustituible, desde su diferencia, su otreidad. Eso es, precisamente, el derecho a la inclusión.

Por eso creo que tomar los derechos humanos en serio exige reconocer que el primer deber que nos impone la universalidad de esos derechos es la inclusión del otro, cada vez más visible como tal otro y cada vez más próximo a nosotros, más presente entre nosotros - inmigrantes, refugiados, minorías-. Pero no al precio que hasta ahora habíamos puesto a la universalidad, esto es, el vaciamiento de toda identidad diferente en aras del abstracto reconocimiento de quien sólo es persona si se asemeja a ese molde pretendidamente vacío pero hecho a nuestra medida que es el canon occidental. Como ha subrayado S. Benhabib ${ }^{12}$, ya no podemos seguir sosteniendo esa suerte de «universalismo de sustitución», que permite al mismo tiempo presentarse como defensor de los derechos humanos y negarlos a quien no es persona porque no devuelve nuestra imagen en el espejo, la del varón, mayor de edad, occidental, autosuficiente o al menos trabajador, heterosexual, etc., imagen a la que no responden las mujeres, los niños, los nacidos fuera del ámbito de opulencia del Norte, los que se identifican con tradiciones culturales ajenas a la occidental, los que no tienen trabajo o sólo lo obtienen en el mercado informal o clandestino o trabajan en el orden privado (en la casa), los que sostienen otras opciones sexuales, etc.

\footnotetext{
${ }^{12}$ Cf. Benhabib.
} 
Es verdad que la versión habitual del universalismo de los derechos humanos, la que nos propone la tradición primigenia de esos derechos, la liberal, cuenta con una importante aportación liberadora, pues hace posible romper las cadenas que legitimaban la sumisión de los seres humanos en aras del respeto al yugo de una tradición legitimada por el transcurso de los siglos, y en ese sentido es verdad que, como propone el postulado del individualismo moral que constituye el núcleo de la tradición liberal, no hay cultura de los derechos humanos sin ruptura con el carácter sagrado de esta o aquella «identidad» cultural. Pero no es menos cierto que al arrojar el agua sucia, las más de las veces, se echa con ella al niño que había que lavar: En ese discurso se nos habla de seres humanos que, para poder ser reconocidos como sujetos, titulares de los derechos, han de despojarse de las condiciones reales que les permiten existir como tales: del género a la lengua, de la clase o la familia a la tradición cultural. En otras palabras, para obtener el reconocimiento como seres humanos, iguales en dignidad y derechos, han de dejar de ser humanos. De nuevo la clarividencia de Marx en su Crítica de la cuestión judía: «el hombre real sólo es reconocido bajo la forma del individuo egoísta, el verdadero hombre es reconocido solamente bajo la forma del sujeto abstracto». Como se han encargado de denunciar, tras la huella de Marx, un buen número de los defensores del esfuerzo por la emancipación - por citar sólo dos, del MacPherson que ofrece en su Teoría política del individualismo posesivo la crítica a los objetivos de dominación subyacentes a ese discurso, al García Calvo que deconstruye el individualismo ontológico en su Contra el hombre-, el único sujeto real que se corresponde con ese ser humano es hoy el sujeto universal del mundo global. Por eso cierto discurso liberal se empeña en hablar de los seres humanos como individuos - mónadas, denunciaba Marx - y sólo de derechos humanos como libertades individuales, al alcance únicamente de un sector mínimo del Norte que pueden permitirse el lujo de ser mónadas y de pagarse las mercancías en que se han convertido el resto de los derechos, lo que llamábamos derechos sociales.

De ahí la insistencia en que uno de los retos más importantes del universalismo, de tomar en serio los derechos, es el desafío que plantean los flujos migratorios al discurso de los derechos humanos y, por supuesto, a su actual institucionalización. En efecto, la lección más importante que nos ofrece una reflexión que se pretenda mínimamente cuidadosa acerca de la relación entre inmigración y derechos humanos, y más aún en un contexto de euforia por la convergencia europea - que parece haber dejado en segundo término o ad calendas graecas otros objetivos como el empleo, por no hablar de la construcción de la UE como un espacio político que satisfaga las condiciones de legitimidad democrática y permita una «ciudadanía europea» que no sea mero elemento de discriminación-, es la confirmación del viejo lema de Ihering que pone en relación directa los derechos humanos con el ideal emancipatorio, sin renunciar a las exigencias concretas: el Derecho no es otra cosa sino lucha 
por el Derecho, por los derechos. Hoy son los inmigrantes, como se ha apuntado desde muy diferentes posiciones, quienes representan ese límite a la aspiración de sujeto universal de los derechos humanos, precisamente por su condición de desposeídos de todo salvo de su condición de seres humanos, que les permite convertirse en agentes de la lucha por superar esta penúltima barrera a la que se enfrenta el viejo ideal emancipador de los derechos humanos. A ellos cabe aplicar el análisis que Arendt dedicara a los refugiados ${ }^{13}$, desprovistos de todo arraigo, obligados a aceptar consecutivamente toda suerte de máscaras para alcanzar la definitiva, el prósopon que les permita el arraigo. Obligados a probar permanentemente una voluntad de integración que poseen en mayor grado que buena parte de la ciudadanía, al mismo tiempo que se les niegan de hecho los medios para esa integración. Y ese deber no consiste en manifiestos retóricos más o menos cómodos que sirven para acallar nuestra malheur de conscience que algunos minimizan como complejo de verdugo etnocéntrico, con escaso sacrificio (el peso lo llevan quienes han de asimilarse forzadamente, renunciar a su identidad, a su cultura, a los suyos), sino que se concreta en transformaciones probablemente exigentes, complejas, pero fáciles de concretar.

Precisamente es lo que se advierte en el nuevo tipo de conflicto para el que resulta funcional el mensaje de emergencia social que hoy se liga como una amenaza derivada de la inmigración: son concretamente los sectores más desfavorecidos de la población los que experimentan como un privilegio tanto su condición de ciudadanos como la de trabajadores: ya no se trata de una situación de derecho, y por ello el enfrentamiento con quienes pueden privarnos de ella es brutal: vale todo (es decir, las respuestas de violencia propias del racismo, por ejemplo). La coartada es, además, que tal presencia rompe o al menos es una amenaza respecto al mínimo de homogeneidad social imprescindible (no ya de la homogeneidad racial o cultural, sino de la posibilidad misma de que todos los nacionales puedan disfrutar de un mínimo de condiciones materiales: puesto de trabajo estable, prestaciones sanitarias, educativas, asistenciales, etc.). Junto a ello, además, se aduce la puesta en cuestión del «patriotismo constitucional», en la medida en que - se dice- no comparten los valores y principios propios de nuestro ordenamiento jurídico constitucional (y es que, mal que pese a Habermas, resulta muy difícil disociar la dimensión étnico-cultural y la jurídica por lo que se refiere a las reglas de juego básicas). En el fondo de este planteamiento late el eco del mensaje roussoniano que exige desterrar del Estado a quien no acate la religión civil y que se manifiesta asimismo en la inscripción de los galeotes añorada también por Rousseau en el Contrato Social: «obligar a ser libres». La homogeneidad impuesta no es el mejor terreno para la democracia que es un régimen con voluntad de inclusión. Tiene razón Dahrendorf cuando escribe que «la auténtica verificación de la fuerza de los derechos de ciudadanía es la heterogeneidad. El respeto común a los títulos de acceso a los bienes fundamentales, atribuidos a personas

\footnotetext{
${ }^{13}$ Cf. Arendt, 1995. Sobre ello, De Lucas, 1996b, cap. 2.
} 
diferentes por su origen, cultura o credo pone a prueba la combinación de identidad y variedad que es el núcleo de la sociedad civil civilizada... por eso... la ciudadanía no será nunca completa mientras no exista una ciudadanía mundial» ${ }^{14}$. Es lo que advierte Walzer: «Ante la existencia de grupos excluidos, la justicia exige un esfuerzo público para permitir a los miembros de esos grupos volver a entrar en la sociedad y actuar de forma independiente en todas las esferas distributivas. Es precisamente el fin de dos bienes sociales estrechamente vinculados: welfare y educación.» Se trata en definitiva de un deber social que no puede confiarse sólo a la sociedad civil, insisto, ni siquiera a lo mejor de ella, a los voluntarios sociales (que son impagables, insustituibles «agencias de inclusión»), porque los sistemas asistenciales mismos dependen de formas organizadas e institucionales de asistencia. El Estado debe facilitar el trabajo de las asociaciones surgidas de la sociedad civil, pero no sólo eso, pues la mejor forma de luchar contra la exclusión es aumentar la cantidad de bienes disponibles, más que redistribuir lo que ya existe, y ello es fruto de decisiones políticas, o, lo que es lo mismo, de modelos de Estado, ahora en que esa discusión es tachada de arqueológica. Por eso me parece justificado concluir que el Estado no puede ser neutral: no puede dejar de comprometerse con el significado de los bienes sociales elementales, pues «lo que caracteriza una comunidad política democrática es el reconocimiento de que todas aquellas transacciones sociales que llevan a los ciudadanos a los márgenes, que producen una clase de excluidos son... siempre injustas» ${ }^{15}$. En otras palabras, eso significa que el Estado no puede abandonar el principio de solidaridad, sino que debe considerarlo, al contrario, como un imperativo, como parte del contenido de la justicia, al igual que lo son la igualdad y la libertad y debe exigirlo de los poderes públicos y de los ciudadanos. Es cierto que el carácter global de la exclusión supera hoy con mucho la capacidad y competencia de los Estados nacionales, pero mientras tanto, mientras llega la hora de la institución de orden global que pueda regular los mercados internacionales para garantizar los derechos humanos de todos los seres humanos, en la lucha contra la exclusión, en el trabajo frente a esa negación elemental de los derechos humanos, el Estado tiene aún mucho que decir.

\section{El límite del reconocimiento de los derechos: la discusión sobre los derechos sociales de los inmigrantes}

Se ha dicho, con acierto (Añón) que los derechos sociales son un test de inclusión. $Y$ es que el término justo para referirse a los inmigrantes, más aún que integrar, o incluso que el propuesto por Halper y Zapata, acomodar, es este de incluir. Pero podría pensarse que si definimos la inclusión, como lo

\footnotetext{
${ }^{14}$ Dahrendorf, art. cit., p. 15.

${ }_{15}$ Walzer, art. cit., p. 113.
} 
he hecho, en términos de empowerment, el test de esa inclusión no lo constituirían tanto los derechos sociales (los sociales, los económicos y culturales), sino en realidad los políticos, los de participación política, que obviamente no se reducen al sufragio activo y pasivo. Pero no es así. Desde luego que los derechos políticos son el último escalón de ese tipo de inclusión, pero los derechos sociales son decisivos porque constituyen una condición sine qua non de la misma. Como ha explicado Amartya Sen, la pobreza no es otra cosa que la disminución general de las posibilidades vitales. Los derechos sociales son la palanca más importante para combatir esa disminución, es decir, para situar a quien es su titular, al menos, en una posición que le abra otras posibilidades vitales, las acordes con el desarrollo libre y equitativo de todos los seres humanos a partir de las capacidades de cada uno de nosotros, y no de las barreras no elegidas, sino impuestas a buena parte de ellos. Por eso hablamos de derechos económicos, sociales y culturales y hablamos también de desarrollo humano, cuyos indicadores van mucho más allá del mero desarrollo económico. Pero semejante planteamiento sólo puede hacerse si se rebasa la óptica neoliberal (la del liberalismo económico, no la del mejor liberalismo, el de Mill, por ejemplo, pues, como ha mostrado Garzón, en esa perspectiva la intervención social no es una herejía, sino un corolario), tal y como propone la noción de ciudadanía social (Marshall) propia del modelo del Estado social o, al menos, el del Estado del bienestar que hoy hemos dado por digno de un rápido entierro.

Lo repetiré. Cuando hay más posibilidades vitales, para empezar, el hecho de inmigrar no se convierte en una necesidad, en la única salida a una situación desesperada, sino en una libre elección, y lo cierto es que hoy no lo es. Pero una vez que el inmigrante ha accedido al país de destino, su margen de posibilidades vitales pasa de nuevo por el grado de reconocimiento y garantía efectiva de los derechos sociales. La realidad, en nuestro país, es que tampoco se produce semejante reconocimiento y garantía, al menos no en el standard que sería exigible, que, a mi juicio, es el de equiparación con los de los nacionales. Y esto no lo propongo sólo desde una perspectiva de moral universalista fácilmente descalificable por utópica, irreal, ingenua, irresponsable. No: basta leer documentos oficiales de la propia Unión Europea, como el Dictamen 365/2002 del Consejo Económico y Social Europeo, al desarrollar el concepto de integración cívica, o la Comunicación a la Comisión Europea (COM 757) del Comisario de Justicia e Interior A. Vitorino de noviembre de 2000.

Para explicar por qué sucede esto, por qué no se reconocen y garantizan eficaz y equitativamente los derechos sociales de los inmigrantes en relación con los de los nacionales, hay que empezar - y es lo que haré en estas pocas páginas - por desmontar algunos tópicos a propósito de los derechos sociales y en particular cuando son derechos sociales de los inmigrantes. Dicho con Ferrajoli, para explicar la «debilidad política» que aqueja a los derechos sociales y los hace objeto de restricciones o de eliminación de la categoría de derechos 
a garantizar por los poderes públicos, hay que explicar su pretendida «debilidad teórica». Los tópicos que niegan a los derechos sociales en su conjunto el carácter de derechos humanos fundamentales en igualdad con los derechos civiles. Porque si los derechos sociales no son tales derechos, sino aspiraciones de mejora, o, más claramente, bienes o servicios (digámoslo ya: mercancías) que en todo caso hay que ganar (merecer, dicen otros que gustan de los eufemismos) con el propio esfuerzo, están sujetos al juego del mercado, cuya lógica es distinta de la de los derechos humanos. Quienes se empecinan en sostener semejante retórica, pues de retórica se trata y no de pragmatismo, como pretenden, ignoran lecciones elementales de la teoría contemporánea de los derechos humanos.

Como se ha argumentado hasta la saciedad (Ferrajoli, Eide, Pérez Luño, Peces-Barba, Prieto Sanchís, Añón, Baylos, Canotilho, Curtis, Abramovich, Contreras, Sastre, etc.), la pretendida distinción teórica entre estos derechos y las libertades negativas (mal llamadas «libertades baratas») no responde a la estructura conceptual de unos y otros, sino a un prejuicio ideológico, propio de los defensores del modelo del Estado mínimo. En el sistema internacional de derechos humanos puesto en marcha en el marco de la ONU (la declaración del 48, los Pactos del 66 y los Convenios de alcance universal) esa tesis de equiparación, junto a la de la interdependencia de unos y otros derechos, es firme. Si eso es así, los derechos sociales no sólo son reconocibles universalmente al igual que los otros, sino que resultan exigibles, en particular en vía jurisdiccional. Y lo interesante, como han mostrado Curtis y Abramovich, cuyo trabajo constituye una de las más relevantes aportaciones a la garantía efectiva de los derechos sociales, es que no sólo son exigibles las reparaciones o sanciones por lesiones en esos derechos, sino también por omisión. Más aún, el análisis que realizan esos autores de la jurisprudencia establecida en las Observaciones Generales del Comité de Derechos Económicos, Sociales y Culturales de la ONU, desde 1985 (junto a los de los Tribunales que han de aplicar y sentar interpretación acerca de los instrumentos regionales de derechos sociales, en el ámbito europeo y americano), proporciona un fundamento teórico sólido para su justiciabilidad en el orden interno, en los Estados nacionales que han ratificado los Pactos del 66 y de los Convenios de la OIT, por ejemplo, es decir, ofrece una palanca decisiva para su exigibilidad en la práctica, tanto en vía directa como indirecta.

Claro que no cabe ocultar que en punto a la garantía efectiva de los derechos sociales la competencia —ergo el deber- más importante corresponde a los Gobiernos. Pero aquí es muy importante señalar que en no poca medida los problemas derivan del incumplimiento por parte de los Estados de obligaciones adquiridas al ratificar instrumentos internacionales: y en particular (van Hoof, Eide) obligaciones de respetar, de proteger, de garantizar y de promover, relativas a la adopción de legislación y de medidas inmediatas que garanticen el ejercicio de esos derechos y la remoción de los obstáculos a ese respecto, 
muy destacadamente, la discriminación en su disfrute, por parte de los poderes públicos y de los particulares. Como ha señalado Añón, «el debate sobre derechos sociales y ciudadanía nos conduce hacia uno de los presupuestos tácitos de esos derechos. Me refiero a la aceptación de responsabilidad por las necesidades de bienestar de los ciudadanos. Esta idea, ciertamente, supone plasmar a través de políticas sociales un principio de justicia distributiva: a cada cual según sus necesidades».

Pero si existen esos problemas en relación con el propio concepto, aún son mayores en el contexto de desmantelamiento del Estado de bienestar, $\mathrm{y}$ aún peor por lo que se refiere al reconocimiento de los derechos culturales y a su extensión a los inmigrantes. Ante todo porque no se toman en serio los derechos culturales como derechos. Los derechos culturales están contaminados, como los sociales, de la arremetida seudoliberal contra los derechos económicos sociales y culturales, que están siendo sustituidos gradualmente mediante una estrategia semántica pero también política, por otras nociones. La argumentación sigue el siguiente patrón:

1) El punto de partida es que la crisis del Estado del bienestar evidencia que no se puede fomentar «irresponsablemente» expectativas ilimitadas de satisfacción de necesidades y menos aún de simples deseos que ni siquiera son necesidades, sino el ansia incontenible del ciudadano mal criado, consumidor irrefrenable e insatisfecho.

2) El siguiente paso es negar la universalidad de sus destinatarios, como lo hace, por ejemplo, la tercera vía de Blair: sólo son titulares de esos derechos los que los necesiten responsablemente.

3) A continuación, se niega su carácter mismo de derechos en cuanto no son tales necesidades: no son equiparables a la libertad, a la vida. De inmediato, se rebaja su satisfacción al ámbito de los «servicios sociales», de forma que ya no son un deber exigible frente a los poderes públicos y frente a terceros.

4) Finalmente, se plantea su adquisición como mercancías mediante el fomento de una «actitud de previsión responsable»: hágase planes de pensiones, suscríbase a una mutua de salud...

En relación con los inmigrantes, ha de destacarse, como decía, el particular acoso que sufren los derechos culturales. Si apartamos la educación y el derecho de acceso a la cultura, en sentido genérico, lo demás no son derechos, sino, como dice nuestra Constitución, principios rectores de la vida socioeconómica. Los derrechos culturales en serio plantean en primer lugar el derecho a la cultura, a la identidad cultural y eso es una noción que los seudoliberales no están dispuestos a aceptar. No como derecho, porque les suena a colectivo, tremenda herejía, pues sólo los individuos (y los individuos como islas, nada de dimensión social) son sujetos de derechos. Además, eso de la identidad cultural, como la lengua, son la crema del pastel, nada de necesidades básicas o bienes primarios. Y ésta es la cuestión: los seudoliberales nunca creyeron 
en la cultura como un bien primario. Su noción elitista de cultura es coherente con ese rechazo del derecho de todos a la cultura. En la disyuntiva, mejor optar por calificar la cultura como algo tan sublime que no puede ser un derecho.

Por consiguiente, peor lo tienen aún quienes reivindican su identidad cultural. Recordaré el viejo chiste que se cuenta a propósito de la respuesta de un paisano a uno de los predicadores evangélicos (no sé si Blanco White) en España en el XIX: si no creo en nuestra religión, que es la verdadera, icómo voy a creer en la suya? Si nuestra cultura que es la única verdadera, no es un derecho, ¿cómo lo va a ser lo de otros. que no es cultura sino barbarie? Aquí hay dos problemas: el etnocentrismo que conjuga la cultura sólo en singular y jerarquiza todo lo demás que sólo se aproxima a cultura si se acerca a nuestro canon, y la presentación de cualquier diferencia cultural como patología. Con eso basta para el fobotipo: las diferencias culturales son un peligro para la universalidad de los derechos humanos.

Eso es, en primer lugar, una bobada. Peligro para los derechos humanos comportan buena parte de las prácticas e instituciones culturales de casi todas las culturas: baste pensar en la construcción de la mujer en nuestra cultura, es decir, en el sistema sexo/género. $O$ en la violencia doméstica, o en la esclavitud. Pero no sólo es estupidez, sino también cinismo: es rentable políticamente sacar a pasear al agresor externo (ablación, velo, sacrificios humanos y lo que haga falta) para exhibir nuestra legitimidad y conseguir reforzarla. Y lo que es peor, supone un ejercicio de aquello que se pretende combatir, el fundamentalismo cultural, porque semejante posición implica admitir la calificación de la nuestra como la mejor (si no la única) cultura; aún más, la única compatible con la democracia y los derechos humanos, y ello además desde una noción de cultura esencialista, cerrada, estática, es decir, lo contrario de lo que sabemos hoy que es toda cultura: negociación, apertura, evolución.

Ésos son los puntos de partida desde los que debemos enjuiciar, en mi opinión, el grado de respeto de los derechos sociales (económicos, sociales, culturales) de los inmigrantes. Claro que alguien podría decir que está por demostrar la extensión de la titularidad de esos derechos precisamente a los inmigrantes. Y a esa objeción basta con responder -en los límites de esta nota - que se trata de una extensión análoga en su universalidad a la que reconocemos respecto a los derechos civiles y, además, que en lo que se refiere a los inmigrantes, ese principio debe reforzarse por la consideración del modelo de ciudadanía cívica o integración cívica que postula para los inmigrantes residentes con carácter permanente la equiparación en el status jurídico político con los nacionales.

Pues bien, si aceptamos ese punto de partida, la valoración que merece un marco jurídico como el español, el que institucionalizan la LO $8 / 2000$, el RD 864/2001 de ejecución de esa Ley y el programa GRECO, en el que los derechos sociales son regateados a los inmigrantes y en algunos casos pura 
y simplemente escamoteados, negados, es que no es un programa de inclusión, ni aun de integración, sobre todo si entendemos ésta en los términos de «integración cívica» que propone para los inmigrantes el mencionado Dictamen 365/2002 del Consejo Económico y Social Europeo o el de «Ciudadanía cívica» del que habla también la COM 757/2000. Aún más, a juicio de muchos de nosotros, ese marco vulnera principios básicos de legitimidad, al infringir lo establecido en el artículo 10 de la Constitución española de 1978, que incorpora a nuestro ordenamiento jurídico instrumentos jurídicos internacionales como los Pactos de 1966, o la Convención de Roma de 1950, o los Convenios de la OIT núms. 87, 11 y 141 en los que se reconocen derechos como los de libertad de asociación, manifestación, sindicación o huelga, por citar tan sólo cuatro. En aplicación de los Convenios mencionados de la OIT, ratificados por España, no cabe respecto a estos dos últimos, por poner un ejemplo claro, discriminación relativa al carácter irregular de los trabajadores inmigrantes. Pero si a ello añadimos el vaciamiento o si se prefiere el notable recorte del derecho a la reagrupación familiar en virtud de lo dispuesto en el artículo 18 de la LO 8/2000 y los artículos 41 y 44 del mencionado RD 864/2002, está claro que los derechos sociales de los inmigrantes (insisto: no sólo de los trabajadores inmigrantes) no son el objetivo prioritario de la política española en materia de inmigración. Y si esto es así, icómo no van a producirse discriminaciones de hecho en el ejercicio de los derechos en cuestión por parte de los particulares? ¿Cómo no se va a producir una discriminación en el acceso al empleo, en el salario, en las condiciones de acceso a los servicios de la Seguridad Social? Y ante la pregunta acerca de los medios eficaces con los que los poderes públicos persiguen esas prácticas (lo que exigiría que los inmigrantes estén en condiciones de denunciarlas, premisa que resulta irreal en tantas ocasiones), la respuesta no puede ser positiva. Lo que es aún peor, se han denunciado, como se subraya en todos los informes realizados por organizaciones independientes, como el presente de SOS Racismo, discriminaciones o, simplemente, denegaciones del disfrute del derecho a la salud, a la vivienda, a la educación.

Recientemente Norman Birnbaum ha recordado a propósito del drama palestino un lema de Lévinas: es imposible dominar al otro sin perder la propia alma. Pues bien, la idea de dominación es la clave de nuestra política de inmigración: no queremos controlar (ni regular), sino dominar los flujos migratorios, como se domeña, es decir, como se domestica un animal de tiro o de monta o un esclavo que nos son útiles, o un juguete que nos da placer o nós entretiene. Queremos dominar la inmigración para evitar riesgos (el de perder nuestro privilegiado status, que a su vez no puede mantenerse sin inmigración), pero sobre todo para autoafirmarnos como dueños, como sujetos, y eso significa establecer como regla en nuestras relaciones con los flujos migratorios una única, la de nuestro propio y exclusivo beneficio, ya sea en términos simples de utilidad o explotación, ya en los más complejos que invocan nuestra 
propia supervivencia, la de nuestro modo de vida iy que no nos lo toquen ni modifiquen ellos!

Reconozcamos, pues, que los derechos sociales son el territorio más inmediato (aunque menos evidente que los políticos) de ese proyecto nuestro de dominación, de construcción de los verdaderos otros, los inmigrantes, como un otro inferior, que convive con nosotros como instrumento, como mal necesario.

\section{El inmigrante como sujeto del espacio público: ciudadanía e inmigración}

La progresiva percepción de las nuevas características de los flujos migratorios y en primer lugar de su carácter estructural, de sus demandas y, desde luego, de los conflictos que comportan y los medios para gestionarlos, obliga a otra mirada sobre la inmigración. Esa mirada exige reconsiderar no pocas categorías de nuestro orden social, jurídico y político y, en particular, una sólo asoma tímidamente, pese a que, en opinión de muchos de nosotros, tiene un carácter decisivo. Me refiero, claro, a la cuestión de la ciudadanía, entendiendo por tal la condición de pertenencia, la cualidad de miembro de la comunidad política, que supone la titularidad de la soberanía y la atribución de derechos que van más allá de los derechos humanos fundamentales. $\mathrm{O}$, como propone Marshall, la condición de quien es miembro pleno de la comunidad política (una condición para la que existen dos cauces, la pertenencia a la comunidad nacional y la presencia en el mercado, cauces que se revelan como factores de desigualdad).

La ciudadanía es un status formal, pero también un vínculo de identidad $\mathrm{y}$, sobre todo, un título de poder. Y la nueva realidad de los flujos migratorios pone en cuestión que los elementos que nos permiten definir quién y por qué es ciudadano y practicar así una discriminación justificada respecto a quienes no lo son, esté, de verdad, justificada, sea aceptable e incluso, simplemente, viable en un mundo en desplazamiento (Naïr), marcado por un proyecto globalizador que dice superar las fronteras. Dicho de otra manera, el debate sobre la inmigración, como se viene insistiendo desde un sector aún minoritario, no puede ser minimizado, pero no porque constituya el gran riesgo, la gran amenaza para la subsistencia del modelo de democracias acomodadas del norte, sino porque es el «escenario» más claro en el que se dirime hoy la vieja cuestión de la democracia, del acceso y la distribución del poder, sobre todo por parte de quienes aparecen como diferentes o, para ser más exactos, para aquellos cuya presencia entre nosotros nos hace comprender que no hemos tomado en serio la condición de la diferencia, que es constitutiva de nuestra propia realidad social a la que aún queremos seguir viendo como homogénea.

En otras palabras, la realidad de los flujos migratorios nos enfrenta a la necesidad de transformar esa condición de ciudadanía que se ha convertido en un instrumento de exclusión (Walzer, Balibar), en un privilegio incompatible 
con la legitimidad democrática a cuyo núcleo pertenece la universalidad de los derechos humanos (Ferrajoli), para poder superar los efectos negativos del modelo de ciudadanía sobre el objetivo de integración política, de empowerment en términos igualitarios (Phillips). Transformar las diferencias entre contrato de ciudadanía y contrato de extranjería o de inmigración, para ser más precisos.

Para finalizar, me propongo presentar - recordar - algunos argumentos que justifiquen por qué la revisión del estatuto de ciudadanía constituye un imperativo ineludible en términos de política de inmigración e incluso, más aún, en los de la democracia plural propia de sociedades que son y serán cada vez más multiculturales, entre otras razones, como consecuencia precisamente de los nuevos flujos migratorios. Pero quizá previamente debería tratar de explicar por qué no se considera así, por qué la opinión dominante no incluye la revisión de la ciudadanía como un ingrediente básico de la gestión de los flujos migratorios.

Las razones por las que todavía hoy la ciudadanía no forma parte de esa agenda política en torno a la inmigración son sin duda complejas. A mi juicio, la más decisiva es, como ya apunté, el hecho de que la visión dominante acerca de la inmigración prima una concepción instrumental, funcional a su vez para una concepción monista de la política, arcaica pero muy arraigada, la propia del Estado nacional (y del sistema de mercado), que escinde el mundo en la dicotomía comunidad política nacional versus todo lo demás, ciudadanos (nosotros, los miembros de la comunidad nacional que lo somos por ello de la comunidad política) frente a extranjeros.

Insisto: la visión instrumental de la inmigración es un corolario de esa concepción política y por eso, lógicamente, no puede plantearse la cuestión de la ciudadanía como contenido de la política migratoria. Esta visión encierra la discusión relativa al modelo político de gestión de la inmigración en un debate acerca de los pros y contras de la «apertura» de nuestras sociedades a esos movimientos migratorios que nos tienen por destino, un debate reducido a un ámbito sectorial, el del mercado (que en tantos planteamientos suplanta a la sociedad) y aún más concretamente, el mercado de trabajo, en una especie de operación contable en la que inevitablemente se busca cómo asegurar el saldo positivo de un juego de oferta y demanda. Esa visión instrumental viene exigida por la ficción de que la presencia del extranjero, del inmigrante (y con ello su status jurídico y político, lo que podríamos llamar el «contrato de extranjería» en relación con el de ciudadanía) es provisional, parcial, superficial. Por tanto, que el mundo, jurídica y políticamente hablando, se divide en dos: los ciudadanos y los que no lo son. Es la visión exigida por una noción de comunidad política, como he propuesto alguna vez, presa del viejo molde de Procusto que identifica el demos como etnos y a su vez presenta éste como homogéneo, sin fisuras, ajeno a lo plural. La conclusión inevitable es que la cuestión de la ciudadanía, por definición — por naturaleza - es ajena al debate 
de la inmigración, en la medida en que ésta no es sino el escenario actual que adopta la vieja cuestión de la extranjería. El inmigrante no puede aspirar al contrato de ciudadanía, sino a otro contrato, provisional, parcial, transitorio y evidentemente inferior, el de extranjería/inmigración.

Que se trata de una visión instrumental lo prueba el carácter meramente coyuntural de los argumentos utilizados tanto por parte de quuienes, a la búsqueda de ese saldo favorable, apuestan por una política de inmigración en la que priman los objetivos de control y rentabilidad interna, como por quienes, con idéntico propósito, abogan por una apertura de fronteras. El quid del balance se centra casi exclusivamente en la determinación del umbral de tolerancia de nuestros mercados y nuestras haciendas públicas (y sólo secundariamente de nuestras calles, escuelas y hospitales), ante la presencia de los inmigrantes. Por eso, la regla que rige el juego, en el fondo, es la misma: admitir lo que necesitamos. La inmigración es una cuestión de reposición, de reemplazamiento de lo que nos falta: Mano de obra en determinados sectores y períodos de tiempo, nacimientos que equilibren la desproporción de clases pasivas y población activa y nos ayuden a mantener la población que necesitamos para contar con una posición en la UE, por ejemplo. La consecuencia es una discusión que atiende exclusivamente a los límites, o, para ser más exactos, al criterio que permita establecer el cupo exacto de inmigrantes que cubran esas necesidades (laboral y/o demográfica).

Casi como en el viejo dicho, podríamos concluir que la diferencia es la misma de la tópica caracterización del optimista y el pesimista ante la botella de vino: medio vacía ya, o medio llena aún. Para reconocer los matices, habría que añadir que, en un caso, los esfuerzos se centran en evitar el desbordamiento, en contener la amenaza de «barca llena», sobre todo ante el pavor que ocasiona el mítico «efecto llamada» que provocarían las irresponsables políticas aperturistas. Por eso, el modelo de inmigración acorde con este planteamiento es el de establecimiento de cupos de trabajadores, según la concepción de inmigración de ida y vuelta. El inmigrante que se desea (y subrayo que la óptica es ésa, unilateral: nuestra visión de la inmigración en la que el otro, sus necesidades, sus proyectos, no cuentan salvo que coincidan con lo que nosotros buscamos y en la que el otro es sobre todo otro homogéneo, el inmigrante, ignorando la diversidad y complejidad de los individuos y de los grupos humanos que inmigran, que es, como mínimo, tan importante como la nuestra) es la generalización del modelo del trabajo de temporada o, como mucho, del viejo modelo alemán del Gastarbeiter. Eso se completa, coherentemente, con una obsesión securitaria para evitar el desbordamiento, para garantizar ‘que sólo recibiremos aquellos que «necesitamos». De ahí, insisto, el que se presente como única respuesta o solución el modelo de cuotas o contingentes y la obsesión por protegerse de cualquier otra forma de inmigración (en primer lugar, el reagrupamiento familiar, pero también la inmigración por razones «humanitarias» $y$, sobre todo, la inmigración que viene precisamente en busca de trabajo y no con el contrato de trabajo bajo el brazo). 
En el otro, en cambio, se sostiene que necesitamos muchos más inmigrantes, porque los empleos que ocupan no son cubiertos por los nacionales, o porque la baja tasa de natalidad vacía el contingente de población activa y hace insostenible el modelo de pensiones. Se apuesta así por una política más flexible en los cupos o incluso por una presencia estable de los inmigrantes entre nosotros, con acentos humanitarios e inequívocamente paternalistas, que no ocultan el mantenimiento de la vieja concepción de la política a la que me he referido. Ahora se trata de «tratarlos bien», de respetar los derechos que tienen como seres humanos (un enorme gesto de progresía este de tomar en serio lo que venimos proclamando como obvio), incluso de reconocerles algunos derechos en los mismos términos de los ciudadanos, pero, por supuesto, dentro de un orden: porque si reconocemos que pueden tener los mismos derechos que los ciudadanos plenos, icómo vamos a mantener la distinción?

A mi juicio, la cuestión de fondo es ésta: mientras la política de inmigración gire en torno a un cálculo unilateral, a lo más que puede aspirar el inmigrante es a ser admitido entre nosotros como un trabajador, asimilado a las condiciones de los trabajadores indígenas, de los nacionales. Su horizonte es, en el mejor de los casos, romper con la doctrina de la «preferencia nacional» que permite una discriminación supuestamente justificada en el status de los trabajadores según su origen. Pero en realidad nunca llega a alcanzar el lugar de los trabajadores nacionales, casi como en el viejo apólogo de Aquiles y la tortuga: lo que le concedemos al inmigrante como trabajador es lo que hace más de cincuenta años arrancaron los movimientos sindicales, no lo que les reconocemos hoy. El inmigrante arrastra el estigma de haber nacido mal, a destiempo, en el lado malo de la geografía. Por eso lo que nunca se plantea es la verdadera integración, la que corresponde a quien no vive entre nosotros provisionalmente, parcialmente, sino a quien quiere ser uno de los nuestros, sólo que de un nosotros plural.

Porque ésta es la lección o, mejor, el desafío de la inmigración: la lógica, la coherencia de los nuevos flujos migratorios obliga a plantear como una secuencia - sin solución de continuidad, aunque con los controles que sea razonable exigir- como reza un trabajo del colectivo IOE, el hilo que une las condiciones de inmigrantes, trabajadores, ciudadanos. Para los inmigrantes, como para los trabajadores hace un siglo, la única oportunidad de acceder a la condición de miembro de la comunidad política es la de convertirse en trabajadores equiparables a los nacionales. Para ser más exactos, en un tipo de trabajadores, pues los que realizan su actividad de modo precario, o en sectores ajenos al mercado de trabajo formal, no gozarán siquiera de esa oportuniđad. El problema es, de un lado, la carrera de obstáculos que separa al inmigrante de la condición de trabajador inmigrante $\mathrm{y}$, después, de la de trabajador. El problema, aún más, es que ni siquiera cuando se accede a esa condición de trabajador se tiene la llave de acceso a la ciudadanía.

Explicar en qué condiciones y con qué contenido puede hablarse de un modelo de ciudadanía que permita la inclusión de los inmigrantes desborda 
las posibilidades de estas páginas y, a buen seguro, mi capacidad. Probablemente exigiría un debate a fondo sobre el modelo de ciudadanía diferenciada como propuesta más apta para albergar esa nueva ciudadanía, inclusiva y parcial. Me limitaré a apuntar algunos elementos, inspirados ampliamente en los trabajos de Ferrajoli, Balibar y Castoriadis sobre las insuficiencias del modelo monista de ciudadanía, pero también en los análisis de Taylor y Walzer sobre ciudadanía e inclusión. Y apuntaré algunas propuestas que, paradójicamente, traen su fuerza del aún balbuciente proceso europeo.

La primera condición es el abandono de la visión instrumental de la inmigración y, con ello, el reconocimiento de que la representación del mundo que permite seguir planteando la presencia de los inmigrantes como un factor ajeno y secundario respecto al vínculo social y al contrato de ciudadanía es insostenible. Reconocer la diversidad y complejidad de la inmigración, su carácter estructural y global, obliga a revisar la simplista dicotomía que permitía considerar la ciudadanía como un coto vedado para quienes no son originarios de la comunidad estatal-nacional ni pueden llegar a serlo porque sólo interesan en su función de productores o, para ser más exactos, como mercancías. Sólo como tales pueden superar las fronteras, pero eso quiere decir que su presencia entre nosotros no alcanza la plenitud de su condición como seres humanos, menos aún como agentes del espacio público.

La segunda, que la necesaria transformación del modelo excluyente y monista de ciudadanía en uno plural e inclusivo, pasa por una redefinición del proceso de integración y del vínculo de ciudadanía. Respecto a lo primero, como he avanzado en otros trabajos, creo que es importante insistir en que el proceso de integración no puede ser visto sólo ni aun primordialmente como el mágico resultado de políticas de interculturalidad, tolerancia y pluralismo, unidas a medidas concretas en los factores básicos de integración: escuela, vivienda y trabajo. Eso es imprescindible (con los matices que se quiera), pero no es suficiente e incluso puede constituir una mera coartada si no hay pasos claros en el primer terreno de la integración, la igualdad en los derechos. Es preciso dejar muy claro qué derechos son derechos de todos, ergo de los inmigrantes también: derechos civiles, claro, pero también derechos sociales, económicos y culturales y derechos políticos, como trataré de señalar. La cuestión es precisar cuáles y por qué entre los derechos que constituyen el triple contenido de la ciudadanía, es decir, las libertades negativas o seguridad jurídica -la garantía del Estado de Derecho-, los derechos sociales - la garantía del Estado de bienestar-y los derechos políticos que hagan real la comunidad libre de iguales :-la garantía del Estado democrático-, pueden ser extendidos en el nuevo contrato de ciudadanía a los residentes.

Respecto al acceso a la ciudadanía, el paso más claro es la necesidad de transferir el vínculo de ciudadanía desde la nacionalidad (también en el sentido de la identidad cultural) a la residencia, y no necesariamente en los términos de la obtención de la residencia permanente como nueva llave de la ciudadanía 
(aunque fuese de una ciudadanía parcial, siempre que ésta no sea entendida como un status definitivo, sino provisional), sino de forma gradual, comenzando por la ciudadanía en la ciudad, por el ámbito municipal.

\section{NOTA BIBLIOGRÁFICA}

AÑón (2000): «El test de la inclusión. Los derechos sociales», en VVAA (AnTón, A., ed.), Trabajo, derechos sociales y globalización, Barcelona, Icaria, 2000.

Balibar (1994): «Qué significa la ciudadanía europea», Revista Internacional de Filosofia Política, núm. 4/1994.

BAUBÖCK, R. (2001): «Recombinant citizenship», en VVAA, Inclusions and exclusions in European Societies, Routledge, Londres.

Benhabib, S. (ed.) (1996): Democracy and Difference. Contesting the boundaries of the Political, Princeton, PUP.

BenHABiB/CoRnell (eds.): Teoría Feminista y Teoría Crítica, Valencia, Cátedra.

CARENS, J. (2000): Culture, citizenship and Community, Nueva York, Oxford University Press.

Castles, S., y Davidson, A. (2000): Citizenship and Inmigration, Londres, MacMillan.

Colectivo IOE (1998): Inmigrantes, Trabajadores, Ciudadanos, Valencia, Patronat Sud-Nord, Universitat de Valencia, 1998.

De LuCAS, J. (1999): «¿Qué políticas de inmigración? (Reflexiones al hilo de la reforma de la Ley de Extranjería en España)», Tiempo de Paz, núm. 55/1999.

- (2000a): «iHay que cambiar la Ley de Extranjería?», Le Monde Diplomatique.

- (2000b): «El marco juridico de la inmigración. Algunas proposiciones acerca de la necesidad de reformar la Ley Orgánica 4/2000», Jueces para la democracia, núm. 38/2000.

- (2001a): «Sobre las condiciones de la ciudadanía inclusiva (el test del contrato de extranjería)», Hermes, núm. 1/2001.

- (2001b): «Ciudadanía y Unión Europea intercultural», Anthropos, núm. 191/2001.

- (2001c): «Las propuestas sobre política de inmigración en Europa y la nueva Ley de Extranjería 4/2000 en España», en VVAA (CoLOMER, ed.), Emigrantes y estabilidad en el Mediterráneo, Valencia, Nomos/P y Coma, 2001.

- (ed.) (2002): El vínculo social, entre ciudadanía y cosmopolitismo, Valencia, Tirant lo Blanch.

- (2002b): «30 propuestas para una política de inmigración», Claves de razón práctica, núm. 121.

- (2002c): «Seis falacias sobre el multiculturalismo», Temas para el debate, núm. 89.

EUMC (2002): «El Ejido revisited», Equal Voices, Issue 08/2002.

FerRAJOL (1998): Derechos y garantías. La ley del más débil, Madrid, Trotta.

- (2001): El fundamento de los derechos fundamentales, Madrid, Trotta.

HALPER, J. (2002): «Accommodation, Cultural space, contact Zones and the Management of Diversity in situations of Conflict: an anthropological Perspective», Paper en el International Symposium on Inmigration Policies in Europe and the Mediterranean, Barceloa, 2002.

KymLICKA (1995): Multicultural Citizenship, Oxford, OUP (hay trad. cast. La ciudadanía multicultural, Paidós, 1998). 
Martiniello, M. (2001): La nouvelle Europe migratoire. Pour une politique proactive de l'inmigration, Bruselas, Labor.

Naïr, S., y De Lucas, J. (1997): Le Déplacement du monde. Migrations et thématiques identitaires, Paris, Kimé.

Pérez Luño (2001): «Diez tesis sobre la titularidad de los derechos colectivos», texto presentado en el seminario Derechos colectivos, Madrid, Universidad Carlos III, 2001.

PhILliPs (1995): The politics of Presence, Oxford, Oxford University Press, 1995.

Requejo, F. (ed.) (1999): Democracy and national pluralism, Londres, Routledge.

TAYLOR (1999): Acercar las soledades. Escritos sobre el federalismo y el nacionalismo en Canadá (versión de I. Álvarez Dorronsoro. Estudio introductorio de J. de Lucas), Gakoa Liburuak, San Sebastián.

VVAA (Moya Escudero, ed.): Comentario sistemático a la Ley de Extranjería, LO 4/2000 y LO 8/2000, Granada, Comares, 2001.

- (Preuss/Requejo, eds.) (1998): European Citizenship, multiculturalism and the State, Baden-Baden, Nomos Verlagsgesellschaft.

- (Roche/VAN BERKel, eds.) (1995): European Citizenship and social exclusion, Aldershot, Ashgate

- (Zapata, ed.) (2001): «Ciudadanía e interculturalidad», Anthropos, núm. 191/2001.

WALZER (1997): Las esferas de la justicia, México, FCE.

WHITOL DER WENDEN (1999): La ciudadanía europea, Barcelona, Bellaterra.

- (2000): ¿Abrir las fronteras?, Barcelona, Bellaterra.

Young (1990): Justice and the Politics of difference, Princeton, PUP (hay trad. castellana, Justicia y Políticas de la diferencia, Madrid, Cátedra, 2000).

ZAPATA, R. (2002): «iDe qué modo las instituciones públicas deberían acomodar a los inmigrantes? Un marco analítico para el análisis», Gestión y Análisis de Políticas Públicas, núm. 20.

- (2002b): L'hora dels inmigrants. Esferes de justicia i politiques d'acomodació, Barcelona, Temes Contemporanis/Proa. 